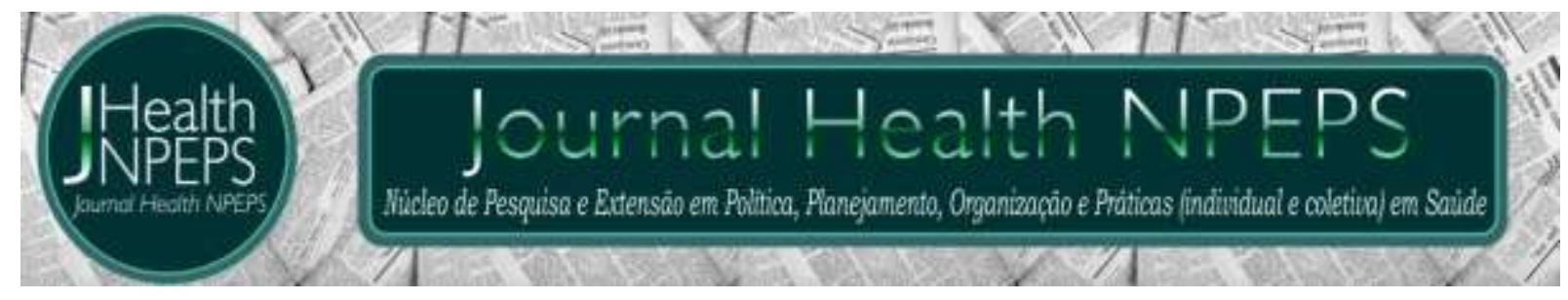

http://dx.doi.org/10.30681/252610103801

ARTIGO ORIGINAL

\title{
Estilos parentales y el involucramiento con el consumo de alcohol en adolescentes de secundaria
}

\section{Parental styles and involvement with alcohol consumption in middle school adolescents}

\section{Estilos parentais e envolvimento com o consumo de álcool por adolescentes do ensino médio}

\section{Mildred Astrid Moreno Cruz ${ }^{1}$, María Magdalena Alonso- Castillo², Nora Angélica Armendáriz-García ${ }^{3}$, Nora Nelly Oliva Rodríguez ${ }^{4}$}

\section{RESUMEN}

Objetivo: determinar la relación de los estilos parentales y el involucramiento con el consumo de alcohol en adolescentes que estudian secundaria. Método: diseño descriptivo-correlacional. Muestra de 389 participantes, se utilizó una cédula de datos personales y de prevalencias de consumo de alcohol y dos instrumentos: escala de estilos de socialización parental en la adolescencia y escala de involucramiento del adolescente con el consumo de alcohol. Resultados: $57.1 \%$ de los adolescentes varones y el $54.6 \%$ mujeres consumieron alcohol en algún momento de sus vidas. Las mujeres tuvieron mayor prevalencia de consumo en el último mes y año. Con respecto a los estilos de crianza con participación en el consumo de alcohol, existe una relación negativa significativa entre el estilo de crianza autoritario del padre ( $r s=-137, p=.007$ ) y ambos padres ( $r s=-.119, p=$ 019), y una relación positiva significativa del estilo negligente materno ( $r s=$ $0.134, p=0.00)$, paterno ( $r s=0.131, p=0.010)$ y ambos $(r s=0.145, p=0.004)$ con participación con el consumo de alcohol. Conclusión: cuanto mayor es la

\footnotetext{
${ }^{1}$ Enfermera. Maestra en Ciencias de Enfermería. Profesor por horas. Universidad Autónoma de Tamaulipas, Facultad de Enfermería de Nuevo Laredo. Nuevo Laredo, Tamaulipas, México. E-mail: milimoreno 16@hotmail.com ORCID ID: https://orcid.org/0000-0001-5295-8817

${ }^{2}$ Enfermera. Doctora en Filosofía con especialidad en Psicología. Profesor de tiempo completo y exlusivo. Líder del Cuerpo Académico Prevención de Adicciones. Universidad Autónoma de Nuevo León, Facultad de Enfermería. Monterrey, Nuevo León, México. E-mail: magdalena alonso@hotmail.com ORCID ID: https://orcid.org/0000-0002-7197-8116. Autor principal - Endereço para correspondência: Av. Gonzalitos \#1500 Nte. Monterrey, Nuevo León, México.

${ }^{3}$ Enfermera. Doctora en Ciencias de Enfermería. Profesor de Tiempo Completo. Universidad Autónoma de Nuevo León, Facultad de Enfermería. Monterrey, Nuevo León, México. E-mail: nordariz@hotmail.com ORCID ID: https://orcid.org/0000-0001-9033-3244

${ }^{4}$ Enfermera. Maestra en Ciencias de Enfermería. Profesor por horas. Universidad Autónoma de Nuevo León, Facultad de Enfermería. Monterrey, Nuevo León, México. E-mail: norisnelly91@gmail.com ORCID ID: https://orcid.org/0000-0001-9299-9891
}

Este artigo está licenciado sob forma de uma licença Creative Commons Atribuição 4.0 Internacional, que permite uso irrestrito, distribuição e reprodução em qualquer meio, desde que a publicação original seja corretamente citada. 
percepción del estilo autoritario, menor es la implicación con el consumo de alcohol, en consecuencia, mayor es la percepción del estilo negligente y la mayor implicación con el alcohol.

Descriptores: Consumo de Alcohol en Menores; Responsabilidad Parental; Consumo de Bebidas Alcohólicas.

\section{ABSTRACT}

Objective: to determine the relationship of parental styles and involvement with alcohol consumption in high school adolescents. Method: Correlational descriptive design. With a sample of 389 participants, a personal data and alcohol prevalence certificate and two instruments were used: parent socialization style in adolescence scale and adolescent involvement with alcohol consumption scale. Results: $57.1 \%$ of male and $54.6 \%$ female adolescents consumed alcohol at some point in their lives. Women had higher prevalence of consumption in the last month and year. With regard to parenting styles with participation in alcohol consumption, there is a significant negative relationship between the father authoritarian style ( $r s=-137, p=.007)$ and both parents ( $r s=-.119, p=019)$, and a significant positive relationship of mother negligent style $(r s=0.134, p=0.00)$, paternal $(r s=0.131, p=0.010)$ and both $(r s=0.145, p=0.004)$ with involvement in alcohol consumption. Conclusion: the greater the perception of the authoritarian style, the lower the involvement with alcohol consumption, consequently, the greater the perception of negligent style and the greater the involvement with alcohol.

Descriptors: Underage Drinking; Parenting; Alcohol Drinking.

\section{RESUMO}

Objetivo: determinar a relação dos estilos parentais e o envolvimento com o consumo de álcool em adolescentes do ensino médio. Método: desenho descritivocorrelacional. Amostra de 389 participantes, com uso de questionário de dados pessoais e de prevalência de consumo de álcool, e dois instrumentos: escala de estilos de socialização dos pais na adolescência e escala de envolvimento dos adolescentes com o consumo de álcool. Resultados: $57,1 \%$ dos adolescentes do sexo masculino e $54,6 \%$ feminino consumiram álcool em algum momento de suas vidas. As mulheres apresentaram maiores prevalências de consumo no último mês e ano. Em relação aos estilos parentais com envolvimento com o consumo de álcool, existe uma relação negativa significativa entre o estilo parental autoritário do pai $(r s=-.137, p=.007)$ e de ambos pais ( $r s=-.119, p=.019)$, e uma relação positiva significativa do estilo negligente materno $(r s=0,134, p=0,00)$, paterno ( $r s=$ $0,131, p=0,010$ ) e de ambos ( $r s=0,145, p=0,004)$ com envolvimento com o consumo de álcool. Conclusão: quanto maior a percepção do estilo autoritário, menor envolvimento com o consumo de álcool, consequentemente maior percepção do estilo negligente maior envolvimento com o álcool.

Descritores: Consumo de Álcool por Menores; Poder Familiar; Consumo de Bebidas Alcoólicas.

\section{INTRODUCCIÓN}

$$
\text { El consumo de alcohol y su }
$$

impacto nocivo en la población es un tema relevante para la Salud Pública, puesto que no sólo ocasiona daños a la salud y pérdida de años de vida 
saludables, también se aumenta la morbilidad asociada a este consumo, discapacidad, deterioro de la calidad de vida y enormes gastos para el Sistema de Salud ${ }^{1}$. De acuerdo a la Organización Mundial de la Salud cada año se producen 3.3 millones de muertes en el mundo debido al consumo excesivo de alcohol ${ }^{2}$ y una muerte cada 100 segundos en la región de las Américas, además el consumo de alcohol contribuye al desarrollo de 200 enfermedades incluidas las neoplasias, la infección por el VIH/SIDA, diversos trastornos cardiovasculares y accidentes de tránsito 3.

México ocupa el décimo lugar en consumo de alcohol en América Latina, se estima que el $63 \%$ de su población consumidora de alcohol son adolescentes y jóvenes de entre 12 y 24 años de edad, quienes inician su consumo por curiosidad, la invitación de sus amigos, la experimentación, la aceptación de un grupo o por invitación de familiares ${ }^{4}$. De acuerdo a las prevalencias de consumo de alcohol a nivel nacional para el año 2016 se reportó que más de la mitad de los estudiantes de secundaria han bebido alcohol alguna vez en la vida (53.2\%), el 24.3\% de los adolescentes consumió alcohol en el último año y el $8.9 \%$ en el último mes, además el 8.6\% de los estudiantes refirieron consumir alcohol excesivamente (cinco copas o más en un período corto de tiempo en una sola ocasión en el último mes).

Además, la edad promedio de inicio de consumo de alcohol es a los 12.6 años, por lo que el consumo de alcohol a una temprana edad se convierte en el principal factor de riesgo de muerte prematura en los adolescentes, en México. Además, en este grupo poblacional existen cinco veces más probabilidad de sufrir trastornos por consumo de alcohol, cuatro veces más de desarrollar dependencia etílica y casi siete veces más probabilidad de sufrir lesiones como consecuencia de un accidente automovilístico o de una pelea derivada del consumo excesivo de alcohol $^{5,6}$.

Por lo anterior los adolescentes se encuentran más expuestos a involucrarse con el consumo de alcohol por el proceso de transición que experimentan, ya que comienza la búsqueda de una identidad a través de adquirir nuevas conductas, aumenta la socialización con diversos grupos, cuestionan las 
normas sociales establecidas y buscan adaptarse a la sociedad donde el consumo de alcohol es una opción que cada vez se normaliza socialmente, por lo que se convierten en un grupo vulnerable de la población para iniciar dicho consumo ${ }^{7}$.

Por ello los factores de riesgo y de protección para el involucramiento del adolescente con el consumo de alcohol cobran relevancia científica y social razón por la que es importante su estudio. Entre los factores de protección o de riesgo, las variables familiares han recibido una atención especial entre los investigadores, ya que el contexto familiar es el principal núcleo de socialización en la transmisión de valores, actitudes y creencias y estas se convierten en determinantes para el funcionamiento y adaptación de los hijos a la sociedad ${ }^{8}$.

Dentro de la familia se encuentran los estilos parentales (autoritario, autorizativo, indulgente y negligente), los cuales son un factor que hace referencia a la forma en que los padres actúan y se relacionan en la formación de los hijos. Existen aportes en la literatura de cómo se relacionan las dos principales líneas de actuación parental: la aceptación/implicación y la severidad/imposición con el ajuste de los hijos y como el resultado de la combinación de estas dos dimensiones se clasifican los cuatro estilos parentales: autoritario, autorizativo, indulgente y negligente $y$ si estas actuaciones parentales constituyen factores de protección o de riesgo para el involucramiento con el consumo de sustancias como el alcohol?.

En el estilo parental autoritario considera que las normas son la base de la educación y la disciplina utilizando la coerción física y la coerción verbal, se mantiene con los hijos una relación demandante, lejana y poco empática, considera el afecto como un factor secundario. El estilo autorizativo fomenta más el diálogo que la imposición para lograr acuerdos con los hijos, cuando el hijo se comporta de manera incorrecta utilizan la privación de algo significativo o de importancia para él como forma de corregir la conducta. En el estilo indulgente no se identifican con claridad las normas, los padres no toman en cuenta las acciones tanto correctas como incorrectas de los hijos, pero se relacionan con ellos de manera 
afectuosa y por último se encuentra el estilo negligente, el cual se caracteriza por el bajo afecto, la nula clarificación de límites y la indiferencia hacia los hijos ${ }^{9-11}$. Se ha documentado la relación de algunos de los cuatro estilos parentales con el involucramiento con el consumo de alcohol y se ha encontrado que el estilo parental negligente se relaciona con el uso y abuso de alcohol indicando que puede ser un factor de riesgo para que el adolescente inicie el involucramiento, mientras que el estilo autorizativo se ha identificado como un probable factor de protección ${ }^{12}$.

De acuerdo a la revisión de literatura se ha indicado que el estilo parental negligente aumenta el involucramiento con el consumo de alcohol, los problemas relacionados con esté y reduce el control del consumo de alcohol por parte del adolescente, se ha identificado al estilo parental indulgente como factor de protección del involucramiento del adolescente con el consumo de alcohol y al estilo autoritario como factor de riesgo ${ }^{15}$.

Por lo anterior los estilos parentales pueden ser determinantes de riesgo o de protección para el involucramiento del adolescente con el consumo de alcohol y la importancia de indagar en esta temática permitirá en un futuro diseñar y aplicar intervenciones preventivas con enfoque multidisciplinario que fortalezcan los estilos parentales protectores y se disminuya o limite el consumo de alcohol en adolescentes. Por ello, el objetivo del estudio fue determinar la relación de los estilos parentales y el involucramiento con el consumo de alcohol en adolescentes que estudian secundaria.

\section{MÉTODO}

El diseño del estudio fue descriptivo correlacional transversal ${ }^{16}$. El estudio se efectuó en el mes de marzo del año 2019. La población de estudio estuvo conformada por 644 adolescentes estudiantes de una escuela secundaria del área metropolitana de Monterrey, Nuevo León. El muestreo fue probabilístico estratificado por grupo y año escolar y en cada estrato se utilizó muestreo por conglomerados unietápico, la muestra se calculó con el paquete estadístico nQuery Advisor ${ }^{\circledR} 7.0$ para una correlación 
alternativa bilateral, con un nivel de significancia de .05, una potencia del $90 \%$, obteniéndose 321 sujetos, al considerar una tasa de no respuesta de $5 \%$ se determinan 338, posteriormente se define el efecto de diseño de 1.15 (efecto mediano) para un tamaño de muestra de 389 participantes. Para la recolección de datos se utilizó una Cédula de Datos Personales y Prevalencias de Consumo de Alcohol (CDPYPCA), la Escala de Estilos de Socialización Parental en la Adolescencia (ESPA29) y la Escala de Involucramiento del Adolescente con el consumo de Alcohol (EIAA). La CDPYPCA contenía datos sociodemográficos y prevalencias de consumo de alcohol.

El primer instrumento que se utilizó fue la ESPA299, la cual evalúa la percepción que tiene el adolescente sobre la relación que tiene con sus padres. La escala valora la actuación del padre y madre por separado mediante 212 reactivos (106 reactivos para evaluar a papá y 106 reactivos para evaluar a mamá) en 29 situaciones significativas, 13 positivas y 16 negativas. La escala es de diferencial semántico, sin embargo, para facilitar el llenado los autores recomiendan utilizar una escala tipo
Likert que va desde 1 que significa nunca, 2 algunas veces, 3 muchas veces y 4 siempre.

La escala consta de 7 subescalas: afecto, indiferencia, dialogo, displicencia, privación, coerción verbal y coerción física mediante la suma de estas se obtiene una medida global para las dimensiones

de Aceptación/Implicación (afecto, indiferencia, dialogo y displicencia) y la dimensión de Severidad/Imposición (privación, coerción verbal y coerción física), de la combinación de estas dos dimensiones se clasifican los cuatro estilos parentales en autorizativo (alta aceptación/implicación y alta coerción/imposición), autoritario, indulgente y negligente.

La ESPA29 reportó un Alpha de Cronbach de .96 en la dimensión de Coerción/Imposición y de .97 en la dimensión de Aceptación/Implicación en una muestra de 3,030 adolescentes de la Comunidad de Valencia ${ }^{9}$ y un Alpha de Cronbach de .80 en la dimensión de Coerción/Imposición y de .81 en la dimensión de Aceptación/Implicación en una muestra de 1,285 alumnos de secundaria y preparatoria de la ciudad de Monterrey, Nuevo León ${ }^{17} y$ 
en el presente estudio reportó un Alpha de Cronbach de .95 en una muestra de 389 adolescentes de la ciudad de Monterrey, Nuevo León.

El segundo instrumento la $E I A A^{18}$, se compone de 14 preguntas diseñadas para determinar aspectos cuantitativos del uso y abuso del alcohol, evalúa los efectos del alcohol en tres dominios: funcionamiento psicológico, relaciones sociales y las relaciones familiares. La calificación de la escala EIAA oscila de 0 a 79 puntos, la cual tiene punto de corte de 42, lo cual significa que pasando este punto se considera que existe abuso del alcohol. Sin embargo, para un análisis más detallado los autores de la escala han clasificado el involucramiento con el alcohol de la siguiente manera, 0 puntos se consideran al adolescente abstemio, de 1 a 19 puntos como poco uso de alcohol, de 20 a 41 como consumo moderado, de 42 a 57 se indica abuso del alcohol y de 58 a 79 probable alcoholismo.

El cuestionario original se encontraba en el idioma inglés, para ser utilizado en población mexicana se sometió a un proceso de traducción y retraducción por un perito autorizado, el cual lo tradujo del inglés al español y posteriormente del español al inglés nuevamente. Se realizó una comparación de ambos cuestionarios original y traducido verificando igualdad en ellos $y$ posteriormente fue utilizado por Jiménez en el año 2004 reportando un Alpha de Cronbach de .79 en una muestra de adolescentes de la ciudad de Monterrey, Nuevo León, en el Estado de Tlaxcala en el año 2016 reportando un Alpha de Cronbach de .89 en una muestra de 300 adolescentes $^{20}$ y en el presente estudio reportó un Alpha de Cronbach de .94 en una muestra de 389 adolescentes de la ciudad de Monterrey, Nuevo León.

El presente estudio contó con la aprobación de los Comités de Investigación y Ética en Investigación de la Facultad de Enfermería de la Universidad Autónoma de Nuevo León con número de registro (FAEN-M1454) así como, de los directivos de la secundaria donde fue realizado el estudio. Se obtuvo el consentimiento informado de los padres o tutores de los adolescentes y el asentimiento del participante. Se brindó información acerca del estudio a los posibles participantes; se les explicó de forma clara y sencilla el objetivo del 
estudio, los instrumentos de medición, el tiempo de aplicación y se enfatizó en el anonimato $y$ confidencialidad de la información. Finalmente se procedió a la entrega de los instrumentos para iniciar la recolección de información, los autores estuvieron atentos a contestar cualquier duda sobre los instrumentos, al concluir la recolección de la información se agradeció a los participantes.

El estudio se apegó a lo establecido por el Reglamento de la Ley General de Salud en Materia de Investigación para la Salud ${ }^{21}$ se garantizó el anonimato y confidencialidad de la información que proporcionó el participante.

Los datos obtenidos se procesaron mediante el programa estadístico Statistical Package for the Social Sciences (SPSS) versión 21.0. La consistencia interna de los instrumentos se determinó a través del Coeficiente de Alfa de Cronbach. Se utilizó estadística descriptiva para obtener frecuencias, proporciones y medidas de tendencia central. Se realizó la prueba de ajuste de normalidad de Kolmogorrov-Smirnov con Corrección de Lilliefors y al no presentar normalidad las variables se decidió utilizar estadística no paramétrica. Para determinar la relación de los estilos parentales con el involucramiento con el consumo de alcohol se utilizó el Coeficiente de Correlación de Sperman.

\section{RESULTADOS}

De acuerdo con los datos obtenidos el 53.2\% de los participantes correspondió al sexo femenino, la edad de los participantes fue de 11 a 15 años, la edad promedio fue de 13.27 años $(D E=.911)$, la edad de inicio de consumo de alcohol reportada fue de 11.89 años $(D E=$ 1.76). Respecto al grado escolar se reportó mayor proporción en los alumnos de tercer grado (37\%).

En la tabla 1 se reportan las prevalencias de consumo de alcohol en estudiantes de secundaria. En la prevalencia de consumo alguna vez en la vida el 57.1\% (IC 95\% [.49-.64] de los participantes del sexo masculino reportaron este tipo de prevalencia, el 54.6\%(IC 95\% [.47-61]) del sexo femenino también lo informó. El $30.8 \%$ (IC 95\% [.24-.37]) de los hombres consumieron alcohol en el último año, a diferencia de las mujeres con el 33.8\% (IC 95\% [.27- 
$.40]$ ), el 13.2\% (IC 95\% [.08-.18]) de al $5.85 \%$ (IC 95\% [.02-.09]) de las los hombres y el 14\% (IC 95\% [.09-.18]) mujeres.

de las mujeres consumieron alcohol en el último mes y el 7.7\% (IC 95\% [.03-.11]) de los hombres consumieron alcohol en los últimos 7 días respecto

Tabela 1 - Prevalência globral, lapsica, actual e instantánea de consumo de alcohol.

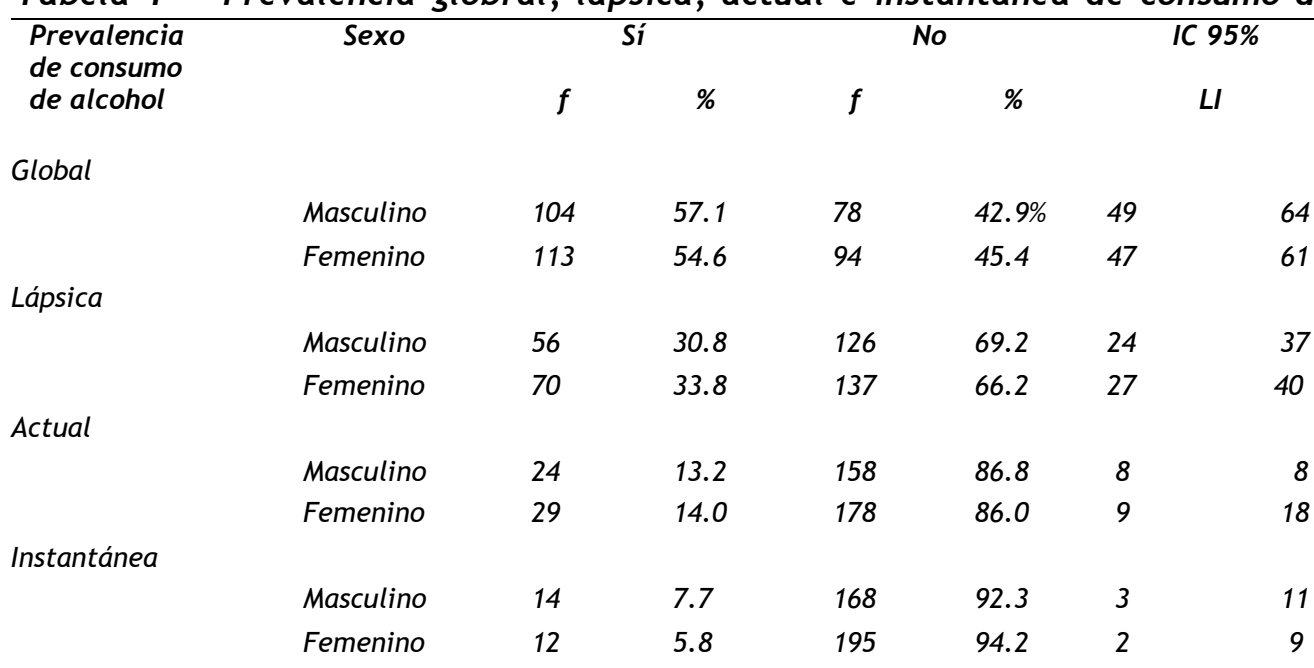

Nota: $f=$ frecuencias, $\%=$ porcentaje, $I C=$ índice de confianza para él \% de respuestas afirmativas, $L I=$ límite inferior, $L S=$ límite superior, $n=389$.

Se puede observar que en las prevalencias alguna vez en la vida, en el último año y en el último mes, fueron más altas en mujeres, no obstante, en la prevalencia instantánea fue más alta en los varones.

En la tabla 2 se observa que el $25.7 \%$ de los participantes perciben un estilo parental indulgente por parte de sus padres, el $29 \%$ un estilo autorizativo, el $21.9 \%$ un estilo autoritario y el $23.4 \%$ un estilo negligente.
Tabla 2 - Frecuencias de Estilos Parentales percibidas.

\begin{tabular}{lcc}
\hline Estilo Parental & $f$ & $\%$ \\
Indulgente & 100 & 25.7 \\
Autorizativo & 113 & 29.0 \\
Autoritario & 85 & 21.9 \\
Negligente & 91 & 23.4 \\
\hline Nota: $\boldsymbol{f}=$ frecuencia, \%= porcentaje, $\boldsymbol{n = 3 8 9 .}$
\end{tabular}

Tabla 3 - Frecuencia de los niveles de involucramiento con el consumo de alcohol por sexo.

\begin{tabular}{lcllll}
\hline Sexo & \multicolumn{2}{c}{ Femenino } & \multicolumn{2}{c}{ Masculino } \\
Involucramiento & $f$ & $\%$ & $f$ & $\%$ \\
Abstemio & 94 & 45.4 & 78 & 42.9 \\
Poco uso de alcohol & 10 & 4.8 & 12 & 6.6 \\
Consumo moderado & 94 & 45.4 & 88 & 48.4 \\
Abuso del alcohol & 9 & 4.3 & 4 & 2.2 \\
\hline Nota: $f=$ frecuencia, \%= porcentaje, $n=389$.
\end{tabular}

De acuerdo a los resultados obtenidos se puede observar que el 
$45.4 \%$ de las mujeres y el $42.9 \%$ de los hombres reportan el nivel abstemio de involucramiento con el alcohol; respecto al consumo moderado se presenta mayor prevalencia en hombres (48.4\%) y se puede observar que las mujeres informaron mayor abuso en el consumo de alcohol con un $4.3 \%$ sobre el $2.2 \%$ reportado por los hombres.

Los resultados que se reportan en la tabla 4, muestran que existe una relación negativa significativa del estilo parental autorizativo paterno $\left(r_{s}=-.137\right.$, $p=.007)$ y de madre y padre en conjunto $\left(r_{s}=-.119, p=.019\right)$ y una relación positiva significativa del estilo negligente materno $\left(r_{s}=.134\right.$, $p=.008)$, paterno $\left(r_{s}=.131, p=.010\right) y$ ambos $\left(r_{s}=.145, p=.004\right)$ con el involucramiento con el consumo de alcohol.

Esto significa que a mayor percepción del estilo autorizativo menor es el involucramiento con el consumo de alcohol y a mayor percepción del estilo negligente mayor es el involucramiento con el consumo de alcohol. Sin embargo, no se encontró relación con los índices autorizativo, autoritario $e$ indulgente.
Tabla 4 - Coeficiente de Correlación de Spearman para los índices de estilos parentales y el índice de involucramiento con el consumo de alcohol.

\begin{tabular}{|c|c|c|}
\hline Variable & $\begin{array}{l}\text { Indice } \\
\text { involucr } \\
\text { con el } \\
\text { de alcol }\end{array}$ & $\begin{array}{l}\text { Imiento } \\
\text { onsumo } \\
\text { la }\end{array}$ \\
\hline $\begin{array}{l}\text { Indice Estilo Autorizativo } \\
\text { (Papá) }\end{array}$ & $\begin{array}{c}r_{s} \\
-.137^{* *}\end{array}$ & $\begin{array}{c}p \\
.007\end{array}$ \\
\hline $\begin{array}{l}\text { İndice Estilo Autorizativo } \\
\text { (Mamá y Papá) }\end{array}$ & $-.119^{*}$ & .019 \\
\hline $\begin{array}{l}\text { İndice Estilo Negligente } \\
\text { (Mamá) }\end{array}$ & $.134^{* *}$ & .008 \\
\hline $\begin{array}{l}\text { İndice Estilo Negligente } \\
\text { (Papá) }\end{array}$ & $.131^{* *}$ & .010 \\
\hline $\begin{array}{l}\text { İndice Estilo Negligente } \\
\text { (Mamá y Papá) }\end{array}$ & $.145^{* *}$ & .004 \\
\hline
\end{tabular}

\section{DISCUSIÓN}

Respecto a las características sociodemográficas de la muestra se documentó que el $53.2 \%$ pertenecen al sexo femenino y el $46.8 \%$ al sexo masculino, la mayor parte de estos estudia el tercer año de secundaria (37.0\%) y la media de edad fue de 13.27años (DE=.911).

Estas características son similares en adolescentes mexicanos que estudian el nivel medio básico conforme a lo que señala el Instituto Nacional de Geografía e Informática en dónde se observa que los hombres y mujeres cuentan con igualdad de oportunidades de integrarse en el nivel de educación básica. En relación con la edad, los datos de estudio son 
similares a los documentados en un estudio realizado en estudiantes de secundaria de Nuevo León donde las edades oscilan entre los 12 y 15 años $^{7}$.

Se reportó según la percepción de los adolescentes que el estilo parental de mayor proporción fue el autorizativo, seguido del indulgente, posteriormente el negligente y por último el autoritario. Lo anterior concuerda con estudios realizados en adolescentes españoles quienes reportaron las mismas proporciones de percepción acerca de los estilos parentales ${ }^{14}$.

Respecto a los resultados se aprecia mayor percepción del estilo autorizativo lo que permite identificar que los participantes perciben de sus padres (ambos) afecto o cariño y se facilita el diálogo ante los cuestionamientos y/ o problemas; no obstante, en menores proporciones identifican que sus padres se comportan hacia ellos indiferentes ante dudas, problemas y conductas utilizando algunas veces el regaño y el castigo como forma de reprimir sus conductas 9 .

Respecto al consumo de alcohol de los participantes, se reportó que la media de edad de inicio de consumo de alcohol fue a los 11.89 años. Estos datos difieren de los documentados por la Encuesta Nacional de Adicciones del año 2016, donde la media de edad de inicio de consumo de alcohol fue de $\bar{X}=16.7$ años; sin embargo, los datos reportados en el presente estudio concuerdan con lo reportado en adolescentes de Nuevo León donde el inicio de consumo de alcohol comienza a temprana edad ${ }^{7}$.

En base a las prevalencias de consumo de alcohol por sexo, se reportó mayor consumo por parte de las mujeres en las prevalencias alguna vez en la vida, en el último año y en el último mes, solo en la prevalencia de la última semana se reportó mayor consumo por parte de los hombres. Los datos anteriores difieren con lo reportado por la Encuesta Nacional de Consumo de Drogas, Alcohol y Tabaco donde el sexo masculino continúa informando mayor proporción en las cuatro prevalencias de consumo de alcohol $^{5}$.

El incremento en la frecuencia de consumo de alcohol por parte de las mujeres podría explicarse en el sentido de que las adolescentes femeninas perciban erróneamente que consumir alcohol en similares 
prevalencias que los hombres es una conducta más de la equidad de género, no obstante, fisiológicamente el consumo de alcohol en cantidades y frecuencias iguales o mayores que los hombres, es mayor el daño en las mujeres por las diferencias metabólicas, bioquímicas y hormonales ${ }^{22}$.

Respecto al objetivo que propuso identificar la relación que existe de los estilos parentales con el involucramiento con el consumo de alcohol se encontraron relaciones negativas significativas entre el estilo parental autorizativo paterno y de ambos padres con el involucramiento con el consumo de alcohol. Además, se documentó relación positiva significativa del estilo negligente materno, paterno de ambos padres con el involucramiento con el consumo de alcohol.

Lo anterior coincide con lo reportado por adolescentes españoles donde los adolescentes con padres que muestran estilos parentales autoritario y negligente reportan mayor involucramiento con el consumo de alcohol que los adolescentes con padres con estilos parentales autorizativos $e$ indulgentes ${ }^{14}$.
Se puede apreciar que al hacer uso del afecto y el razonamiento sobre los regaños verbales y los castigos físicos se pueden establecer mejores relaciones entre padres e hijos, lo cual puede facilitar que se establezca una mejor comunicación con los adolescentes sobre los riesgos y las consecuencias que puede llevarles el iniciar a consumir alcohol a una temprana $\operatorname{edad}^{22}$.

Durante el desarrollo de la aplicación de las encuestas la única limitante encontrada fue el número de reactivos de la escala de socialización de estilos parentales en la adolescencia, la cual cuenta con 212 reactivos y se observó que los participantes tardan alrededor de 25 minutos en contestarla.

\section{CONCLUSION}

En base a los resultados del estudio se identificó que el estilo parental autorizativo se asoció con menor riesgo de involucramiento con el consumo de alcohol y al estilo negligente con un mayor involucramiento con el consumo de alcohol; por lo que sería importante en un futuro implementar 
intervenciones que incluyan a los padres de familia abordando tópicos que fortalezcan los estilos parentales y su efecto en la prevención del consumo de alcohol.

\section{REFERENCIAS}

1. Duffy D. Consumo de alcohol: principal problemática de salud pública de las Américas. Rev latinoam cienc psicol. 2015; 7:371-382.

2. World Health Organization. Global Status report on alcohol and health, 2014. Available from: http://apps.who.int/iris/bitstrea $\mathrm{m} /$ handle/10665/112736/9789240 692763_eng.pdf;jsessionid=56EFE9 111DE50A6DCD9C864C6D1C927D?s equence $=1$.

3. OPS. Informe de la situación regional sobre el alcohol y la salud en las Américas, 2015. Available from:

https://www.paho.org/hq/dmdoc uments/2015/alcohol-Informesalud-americas-2015.pdf.

4. Secretaria de Salud (MX). Aumenta el consumo de alcohol en jóvenes, 2016. Available from: https://www.gob.mx/salud/articu los/aumenta-el-consumo-dealcohol-entre-jovenes.

5. INSP. Encuesta Nacional de Consumo de Drogas, Alcohol y Tabaco: Reporte de Alcohol, 20162017. Available from: https://drive.google.com/file/d/ 1rMlKaWy34GR51sEnBK2-

u2q_BDK9LA0e/view.

6. Villatoro J, Medina-Mora M, Del Campo R, Fregoso $D$, Buslos $M$, Reséndiz $\mathrm{E}$, et al. El consumo de drogas en estudiantes de México: tendencias y magnitud del problema. Salud Mental. 2016; 39(4):193-202.

7. Alonso-Castillo M, Yáñez-Lozano A, Armendáriz García N. Funcionalidad familiar y consumo de alcohol en adolescentes de secundaria. Salud y drogas. 2017; 17(1):87-96.

8. Clayton R. Transitions in drug use: risk and protective factors, vulnerability to drug abuse. American Psychological Association. 1992; 15-51.

9. Musitu G, García F. Escala de Estilos de Socialización Parental en la Adolescencia, ESPA29. Madrid: TEA Ediciones; 2001.

10. Maccoby E, Martin A. Socialization in the context of the family: 
Parent-child interaction. New York: Wiley; 1983.

11. Baumrind D. Current theories of parental authority. Dev psychol. 1971; 4(1Pt2):1-103.

12. Becoña E, Martínez U, Calafat A, Fernández-Hermida JR, Sumnall H, Gabrhelík R. Parental permissiveness, control, and affect and drug use among adolescents. Psicothema. 2013; 25:292-298.

13. Cerutti F, Ramos S, Argimon I. A implicacao das atitudes parentais no uso de drogas na adolescencia. Acta colomb psicol. 2015; 18:73181

14. Martínez I, Fuentes M, García F, Madrid I. El estilo de socialización familiar como factor de prevención o riesgo para el consumo de sustancias y otros problemas de conducta en los adolescentes españoles.

Adicciones. 2013; 25(3):235-242.

15. Souza k, Ribeiro D. Estilos percibidos e o consumo de álcool entre adolescentes. Adolesc saúde. 2016; 13(1): 89-97.

16. Burns N, Grove SK. Investigación en Enfermería. Barcelona: Manual Moderno; 2012.
17. Villarreal M. Un modelo estructural del consumo de drogas y conducta violenta en adolescentes escolarizados (Tesis de Doctorado). Facultad de Psicología. Monterrey NL; 2009.

18. Mayer J, Filstead W. The Adolescent Alcohol Involvement Scale, an instrument for Measuring Adolescent's Use and Minuse of Alcohol. J stud alcohol drugs. 1979; 40:291-300.

19. Jiménez El. Expectativas y abuso de consumo de alcohol en adolescentes.

Repositorio Universidad Autónoma de Nuevo León; 2004.

20. Xancal-Xolaltenco E, TorresReyes A, Morales-Castillo F, Arrioja-Morales G, Ávila-Arroyo M, Tenahua-Quitl I. Factores de Riesgo Psicosocial y su relación con el Involucramiento con el Alcohol en Adolescentes. Opción. 2016; 32(13):255-266.

21. Secretaría de Salud (MX). Ley General de Salud en Materia de Investigación en Salud. Última actualización. México: Secretaría de Salud; 2016.

22. Carbajal A. La nutrición en la red, 2018. Available from: 
https://www.ucm.es/nutricionc arbajal/.

23. Patock-Peckham JA, MorganLópez AA. College drinking behaviors: mediational links between parenting styles, impulse control and alcoholrelated outcomes. Psychol Addict Behav. 2006; 20(2):117-125.

Conflito de interesses: Os autores declaram não haver conflito de interesses.

\section{Participação dos autores:}

- Concepção: Moreno MAC, Alonso-Castillo MM, Armendáriz-García NA, Oliva NNR.

- Desenvolvimento: Moreno MAC, Alonso-Castillo MM, ArmendárizGarcía NA, Oliva NNR.

- Redação e revisão: Moreno MAC, Alonso-Castillo MM, ArmendárizGarcía NA, Oliva NNR.

Como citar este artigo: Moreno MAC, Alonso-Castillo MM, Armendáriz-García NA, Oliva NNR. Estilos parentales y el involucramiento con el consumo de alcohol en adolescentes de secundaria. J Health NPEPS. 2019; 4(2):215-229.

Submissão: 09/07/2019

Aceito: $18 / 11 / 2019$

Publicado: 01/12/2019 